\title{
O processo de metacognição durante uma olimpíada de simulação clínica
}

\author{
The metacognition process during a clinical simulation olympiad
}

El proceso de metacognición durante una olimpiada de simulación clínica

\begin{abstract}
Katy Conceição Cataldo Muniz Domingues ${ }^{1 *}$, Thais Lazaroto Roberto Cordeiro ${ }^{1}$, Gerson Alves Pereira Júnior², Maurício Abreu Pinto Peixoto.
\end{abstract}

\section{RESUMO}

Objetivo: Investigar a presença da metacognição na simulação clínica em ambientes competitivos e descrever o processo de pensamento de uma equipe de atendimento por meio do uso de ferramentas metacognitivas. Métodos: Estudo de caso qualitativo, orientado para a descrição do uso da metacognição na equipe vencedora de uma olimpíada de simulação clínica. A coleta de dados ocorreu em um congresso de educação médica. Utilizou-se como referencial teóricoa Metacognição, fazendo uso das categorias teóricas de Efklides $\mathrm{A}(2008)$ para o processo analítico de dados coletados por entrevistas semiestruturadas em grupo, analisadas pela técnica de análise de conteúdo de Bardin L (2016). Resultados: No discurso da equipe, foram observados 77 episódios de expressão da Metacognição, distribuídos entre Conhecimento (28), Experiência (22) e Habilidade (27). Estes, foram ainda classificados em suas subcategorias a saber respectivamente; Conhecimento sobre a pessoa, a tarefa e a estratégia; Experiência de sentimento de dificuldade, confiança, familiaridade e saber; e Habilidade de previsão, planejamento, monitoração e avaliação. Conclusão: Foi possível perceber a correspondência entre as ações e desempenho da equipe durante a olimpíada e os processos de pensamento, tais como descritos pela teoria metacognitiva. Apontamos argumentos em defesa do papel educacional da metacognição.

Palavras-chave: Metacognição, Educação em saúde, Aprendizagem, Simulação, Ensino.

\begin{abstract}
Objective: To investigate the presence of metacognition in clinical simulation in competitive environments and describe the thought process of a care team through the use of metacognitive tools. Methods: Qualitative case study, oriented to the description of the use of metacognition in the winning team of a clinical simulation Olympiad. Data collection occurred at a Congress of Medical Education. Metacognition was used as a theoretical reference, using the theoretical categories of Efklides $A$ (2008) for the analytical process of data collected by semi-structured group interviews, analyzed by Bardin's content analysis technique of Bardin L (2016). Results: In the team discourse, 77 episodes of metacognition expression were observed, distributed among Knowledge (28), Experience (22) and Skill (27). These were also classified in their subcategories to know respectively; Knowledge about the person, task and strategy; Experience of feeling difficult, confidence, familiarity and knowledge; and Ability to predict, plan, monitor and evaluate. Conclusion: It was possible to perceive the correspondence between the actions and performance of the team during the Olympics and the thought processes, as described by the metacognitive theory. We point out arguments in defense of the educational role of metacognition.
\end{abstract}

Keywords: Metacognition, Health education, Learning, Simulation, Teaching.

1 Universidade Federal do Rio de Janeiro (UFRJ), Rio de Janeiro - RJ. *E-mail: enfkaty@gmail.com 


\section{RESUMEN}

Objetivo: Investigar la presencia de metacognición en la simulación clínica en entornos competitivos y describir el proceso de pensamiento de un equipo de atención mediante el uso de herramientas metacognitivas. Métodos: Estudio de caso cualitativo, orientado a la descripción del uso de la metacognición en el equipo ganador de una Olimpiada de simulación clínica. La recolección de datos ocurrió en un Congreso de Educación Médica. Se utilizó la metacognición como referencia teórica, utilizando las categorías teóricas de Efklides A (2008) para el proceso analítico de los datos recogidos por entrevistas grupales semiestructuradas, analizadas por la técnica de análisis de contenido de Bardin L (2016). Resultados: En el discurso del equipo se observaron 77 episodios de expresión de metacognición, distribuidos entre Conocimiento (28), Experiencia (22) y Habilidad (27). Estos también se clasificaron en sus subcategorías para conocer respectivamente; Conocimiento sobre la persona, tarea y estrategia; Experiencia de sentirse difícil, confianza, familiaridad y conocimiento; y Capacidad para predecir, planificar, monitorear y evaluar. Conclusión: Fue posible percibir la correspondencia entre las acciones y el desempeño del equipo durante los Juegos Olímpicos y los procesos de pensamiento, como lo describe la teoría metacognitiva. Señalamos argumentos en defensa del papel educativo de la metacognición.

Palabras clave: Metacognición, Educación para la salud, Aprendizaje, Simulación, Enseñanza.

\section{INTRODUÇÃO}

O erro médico tem sido uma preocupação continuada na comunidade médica. Por exemplo, estima-se que aproximadamente 98 mil mortes por nos EUA. A incidência de erros de medicação tem variado entre $3 \%$ e $27 \%$ e ainexperiência, tem sido importante fator de risco para danos ao paciente, seja por conta da adoção de um novo procedimento, ou devido à ação de médicos em fase inicial de treinamento (GOMES TR, et al., 2017, MENDONÇA V, et al., 2019).

Etapa relevante para melhorar a qualidade do cuidado é o treinamento dos profissionais de saúde, onde a simulação,consiste em uma metodologia de imersão dos estudantes em cenários similares ao da prática assistencial com o objetivo de treinar e/ou avaliar as habilidades, competências, e atitudes esperadas dos futuros profissionais, além de mitigar dilemas éticos tradicionais da formação médica, ao reduzir a exposição do paciente a estagiários inexperientes(JONES F, et al., 2015; KANEKO RMU e LOPES MHBM, 2019; BRANDÃO CFS, 2018; CARVALHO AB,et al., 2021; ASSOCIAÇÃO BRASILEIRA DE EDUCAÇÃO MÉDICA (ABEM), 2021).

Processos de liderança e comunicação foram observados em equipes de emergência, mostrando-se insuficientes. Autores apontaram que a simulação de alta fidelidade aplicada nestas populações foi útil no desenvolvimento destas habilidades. Além disso, sugerem a implementação de programas de treinamento que possam melhorar a comunicação entre os membros da equipe, assim como a competência do líder em gerenciar as ações dos seus liderados (JONES F, et al., 2015; LINS ML, et al., 2018; CAVEIÃO C, et al., 2018).

Se entendermos a simulação como "imitação de um processo ou sistema do mundo real", então podemos traçar suas origens no passado profundo. O Xadrez, por exemplo, é uma forma de simular processos militares, e tem sido relacionado ao longo dos anos, a diversas habilidades cognitivas ligadas a processos que envolvem o aprendizado, como: memória de trabalho, metacognição, leitura e escrita, raciocínio lógico-matemático, flexibilidade, planejamento, tomada de decisão, concentração, capacidade de analisar, sintetizar e resolver problemas, autocontrole e autoavaliação (ROSA RR, et al., 2020).

No âmbito de ensino em saúde, a simulação começou a ganhar maior espaço e robustez a partir da década de 1960, fortalecendo-se como um processo de instrução (BRANDÃO CFS, et al., 2014; SINZ E, et al., 2021). Este foi o ano inclusive do primeiro manequim de corpo inteiro (Ressusci Annie) (CARVALHO DRS, et al., 2021). 
Dispensa comprovação o fato de que a metacognição se faz presente tanto na vida cotidiana como em atividades de simulação. $O$ que nem sempre acontece é termos consciência dela. $E$ isso importa muito para 0 aprendizado. Aprende-se melhor quando se sabe como pensamos ao fazê-lo. Segundo Peixoto MAP, et al. (2021) conhecer nossas cognições, é o primeiro passo para compreender a Metacognição. A Cognição está diretamente ligada às nossas operações mentais de percepção, atenção, memorização, leitura, escrita, compreensão, comunicação etc.

Metacognição é justamente o conhecimento e gerênciadessas operações mentais; o que são, como se realizam, quando se usa uma em detrimento da outra e que fatores auxiliam ou interferem em sua operação. Em sua definição original, refere-se ao conhecimento que as pessoas têm sobre sua própria cognição e a tudo que os envolvem (FLAVELL JH, 1976). Neste sentido podemos afirmar que seus domínios se estendem também aos processos de autorregulação pelo uso da monitoração e controle da cognição visando objetivos desejados pelo sujeito.

Para Efklides A (2008), a Metacognição é multifacetada, e embora não implique sempre e necessariamente em consciência plena do indivíduo, essas facetas estão relacionadas com o que o sujeito sabe sobre seu processo cognitivo (Conhecimento Metacognitivo - CM), ao seu estado afetivo ou cognitivo (Experiência Metacognitiva - EM) ou ao que ele está fazendo ou precisará fazer (Habilidade Metacognitiva $\mathrm{HM})$.

A ideia de utilizar a simulação como estratégia de aprendizado na educação dos profissionais de saúde, não se restringe a avaliação de conhecimentos técnicos adquiridos, mas revela-se importante aliada para mensurar e analisar comportamentos frente a situações de diversas, como por exemplo: gerenciamento de situações de crise, liderança e relacionamento de equipe (FLATO UAP e GUIMARÃES HP, 2011).

A metacognição já tem sido usada no apoio a atividades de simulação (BRANNICK MT, 2013; DREIFUERST KT, 2015). Reflexões que a metodologia de simulação clínica pode ser capaz de fazer emergir o saber pensar do sujeito, a partir da realidade recriada nos cenários propostos, fornecendo aos alunos a condição de autonomia na tomada de decisão, têm apontado para discussões em torno desta temática.

Em estudo de Peixoto MAP, et al. (2021), demonstrou-se que a metacognição pode revelar erros de raciocínio que levam a diagnósticos médicos equivocados. Embora seja razoável supor sua importância, não se tem investigado o processo metacognitivo em situações competitivas, tais como em olimpíadas.

O presente estudo visa responder a duas questões principais: A metacognição está presente em situações de simulação em ambientes competitivos? É possível descrever o processo de pensamento de uma equipe de atendimento por meio do uso de ferramentas metacognitivas?

Dessa forma, o objetivo foi: Investigar a presença da metacognição na simulação clínica em ambientes competitivos e descrever o processo de pensamento de uma equipe de atendimento por meio do uso de ferramentas metacognitivas.

\section{MÉTODOS}

Trata-se de um estudo de caso com desenho qualitativo orientado para a descrição do uso da metacognição pelos estudantes da equipe vencedora da olimpíada, que ao serem convidados, todos aceitaram a participarda pesquisa. Teve como referencial teórico a Metacognição, fazendo uso das categorias teóricas de Efklides A (2008) para o processo analítico. De modo a preservar o anonimato dos sujeitos, seus nomes foram substituídos porsiglas.

$\mathrm{Na}$ pesquisa qualitativa, as experiências dos indivíduos e suas percepções são aspectos úteis e importantes para a pesquisa. Esta realidade é construída em conjunto entre investigador e investigado a partir das experiências individuais de cada sujeito (Epistemologia) e da realidade múltipla e subjetiva (Ontologia) (PATIAS ND eHOHENDORFF JV, 2019). 
O cenário deste estudo foi a Olimpíada de atendimento a pacientes críticos, instituída como evento précongresso regular em um congresso de educação médica. O evento utiliza cenários de simulação clínica, tendo como base a construção das estações da prova do Revalida (Prova que subsidia o processo de revalidação dos diplomas de médicos graduados fora do Brasil). O período de coleta foi omitido para preservar o anonimato dos sujeitos já que durante a olimpíada só há uma equipe vencedora e nesse congresso de educação médica só há uma olimpíada. Assim, informar o ano significa informar quem são os participantes já que as premiações estão publicamente disponíveis.

Como técnica de coleta de dados, optou-se pela entrevista semiestruturada em grupo), com perguntas fechadas e abertas, oferecendo ao entrevistado mais conforto ao responder livremente às questões propostas (MINAYO MCS, 2010). O grupo foi entrevistado em local privativo e confortável, sendo a entrevista gravada e filmada e posteriormente transcrita.

A entrevista iniciou-se com perguntas genéricas, que progressivamente e norteadas pelos próprios participantes, foram sendo direcionadas para temas mais específicos. As perguntas foram dirigidas na maior parte das vezes, para todos indistintamente. Porém, para detalhar eventos específicos ou explicitar pensamentos, às vezes eram feitas novas perguntas elaboradas pelo entrevistador durante a entrevista.

A entrevista foi submetida à técnica de análise de conteúdo de Bardin $L$ (2016), com realização de quatro etapas sequenciais: Na primeira etapa, a entrevista foi gravada e posteriormente transcrita para arquivo texto. Na segunda, de leitura flutuante, o texto foi lido repetidamente pelo entrevistador para entender o sentido global dos eventos, assim como os papéis particulares dos sujeitos e suas relações no grupo.

$\mathrm{Na}$ terceira etapa, o texto foi codificado com o auxílio do processador de textos word, segundo as categorias metacognitivas de Efklides A (2008). Nesta etapa os sentidos globais percebidos foram sendo traduzidos em termos de variáveis metacognitivas. Isto é, foram atribuídos códigos a trechos das entrevistas identificados como relevantes. Assim, ao final desta etapa havia uma listagem de todos os trechos que evidenciaram atividade metacognitiva, codificados pela variável metacognitiva correspondente.

$\mathrm{Na}$ quarta e última etapa buscou-se, então, descrever sob a perspectiva metacognitiva o potencial da simulação clínica como metodologia de ensino capaz de favorecer o aprendizado dos alunos e a promoção da melhoria de desempenho.

O estudo respeitou as Resoluções 466/12 e 510/16 do CNS, e foi submetido ao Comitê de Ética em Pesquisa do Instituto de Estudos e Saúde Coletiva da UFRJ sob o número CAAE 96007018.5.0000.5286 e aprovado pelo parecer no. 2.938.945. Cabe-nos informar que a ABEM e as Comissões do COBEM, também tiveram ciência prévia e aprovaram a realização do estudo.

\section{RESULTADOS}

Durante a simulação clínica a Metacognição fez-se presente. Foram identificados 77 eventos metacognitivos, definidos como trechos do discurso onde era possível identificar a presença de algum fenômeno metacognitivo. Cada evento metacognitivo foi classificado de acordo com as categorias teóricas fornecidas por Efklides A (2008), conforme disposto (Quadro 1).

Quadro 1 - Eventos metacognitivos observados classificados segundo Efklides A (2008)

\begin{tabular}{|c|c|c|}
\hline $\begin{array}{c}\text { Conhecimento } \\
\text { Metacognitivo (CM) } \\
\mathbf{N}=\mathbf{2 8}\end{array}$ & $\begin{array}{c}\text { Experiências } \\
\text { Metacognitivas (EM) } \\
\mathbf{N}=\mathbf{2 2}\end{array}$ & $\begin{array}{c}\text { Habilidade } \\
\text { Metacognitiva (HM) } \\
\text { N = 27 }\end{array}$ \\
\hline $\begin{array}{l}\text { - Sobre a pessoa } \\
\text { - Sobre a tarefa } \\
\text { - Sobre a estratégia }\end{array}$ & $\begin{array}{l}\text { - Sentimento de dificuldade } \\
\text { - Sentimento de confiança } \\
\text { - Sentimento de familiaridade } \\
\text { - Sentimento de saber }\end{array}$ & $\begin{array}{l}\text { - De previsão } \\
\text { - De planejamento } \\
\text { - De monitoração } \\
\text { - De avaliação }\end{array}$ \\
\hline
\end{tabular}

Fonte: Domingues KCCM,et al., 2021. 
O CM é o conhecimento declarativo armazenado na memória, sobre habilidades e estratégias cognitivas, tarefas e ainda modelos de processos cognitivos. É a crença que a pessoa possui sobre si mesma e a respeito de alguma coisa. Pode ser subdividido em três subcategorias; conhecimentos sobre pessoas, tarefas e estratégias. A subcategoria de pessoas inclui quaisquer conhecimentos e crenças que o sujeito possa adquirir quanto aos seres humanos como processadores cognitivos. A de tarefa, tem relação com a natureza da informação e da atividade. Já o conhecimento sobre estratégias, refere-se ao caminho mais adequado para se obter êxito numa tarefa (FLAVELL JH, 1979; LOCATELLI SW, 2014).

Observou-se que o CM sobre as pessoas conferiu segurança aos participantes em relação ao que cada um possuía de conhecimento sobre o cenário de simulação, conferindo tranquilidade para que a equipe prosseguisse na competição:

$$
\begin{aligned}
& \text { "A gente sabe que todo mundo é capaz" (MC). } \\
& \text { "Já sabemos o bastante sobre esses assuntos" (G). }
\end{aligned}
$$

O CM sobre a tarefa foi outro fator de segurança para a equipe, já que a maioria dos participantes era monitor da disciplina de simulação. Tal fato é percebido claramente na fala dos participantes:

\section{"A gente estuda junto há bastante tempo $(R)$ e "A gente já tem uma vivência com isso, com a simulação clínica" (I).}

O CM sobre a estratégia apresentou-se como ponte entre o CM sobre a pessoa e a tarefa, fornecendo subsídios necessários ao cumprimento da meta. Podemos identificar a consonância entre esses conhecimentos, nas seguintes falas:

"Dentro de um atendimento não tem só as medidas tipo assim, farmacológicas, as ações que devem ser tomadas, mas a forma com que você conduz, então, a questão da calma, a questão da comunicação com a equipe, a efetividade desta comunicação, achei que isso seria muito válido" (MC).

"Eu penso muito em estratégia, no que a gente tem que fazer, a gente tem que formar aquilo e fazer aquilo" (I).

As EM são a interface entre a pessoa e a tarefa, estão relacionadas ao estado de consciência do indivíduo e ao que ele vai sentir quando estiver processando as informações ou realizando uma tarefa. Têm caráter afetivo de valor positivo ou negativo e relacionam-se a diferentes ações e processos cognitivos. Podem ser classificados em sentimentos de dificuldade, familiaridade, saber e confiança. A sensação de saber, por exemplo, representa um sentimento positivo, e reforça na pessoa a segurança relativa ao conhecimento que ela possui sobre um determinado item (EFKLIDES A, 2008).

Percebemos tais experiências ao reparar que o sentimento de familiaridade entre os participantes foi um dos pré-requisitos para a formação da equipe, bem como, no sentimento de confiança no conhecimento de cada nome sugerido:

"Nós todos somos monitores da mesma matéria então em questão de conhecimento e bagagem a gente confia basicamente em cada um" (G).

"Eu ia ficar contente em questão assim, de achar que a equipe tinha uma qualidade intelectual boa" (R).

Também foi possível identificar o sentimento de dificuldade, de valor negativo, percebido em situações de interrupção ou falta de fluência no processamento da tarefa:

"Você acha que não tem a capacidade de responder ou você nunca viu, você se sente um pouco culpada, derrotada" (MC).

As HM foram são processos de pensamento que demonstram a capacidade do sujeito que, ao conhecer como funciona seu raciocínio, usa intencionalmente estratégias específicas para modular o seu processo 
cognitivo. São classificadas em habilidades metacognitivas de previsão, planejamento, monitoração e avaliação. Para serem ativadas, é preciso que haja consciência da fluência de processamento cognitivo e consciência de que um conflito ou erro ocorreu (EFKLIDES A, et al., 1999).

Reconhecemos na fala dos participantes a estratégia de previsão ao observar que existia um determinado tempo para a conclusão da tarefa que se não fosse obedecido, resultaria na eliminação da equipe. Desta forma, a equipe se organizou no sentido de trabalhar mais lentamente as tarefas difíceis e mais rápidas as tarefas mais fáceis:

"A gente tomou as medidas necessárias no momento. A gente não negou o fato e a inalação, na verdade a inalação não deu tempo de fazer efeito" (E).

"A gente tinha quinze minutos, que é o tempo para todos os casos, só que a paciente se você não fizesse a conduta completa, ela morreria antes" (G).

A estratégia de planejamento foi identificada ao percebermos o momento quando, como e porque os participantes agiram para alcançar os objetivos da tarefa. Nas falas a seguir, reconhecemos o pensar com antecipação no sentido de resolver o problema:

"A gente falou, eclâmpsia, aí que falamos, temos que sulfatar com hidralazina” (MC).

"É uma medida geral em crise convulsiva" (R).

A estratégia de monitoração pode ser descrita como uma forma de controle autorregulado de nossas habilidades cognitivas usadas durante o desempenho de uma tarefa, possibilitando a mudança de planos se for necessária (PEIXOTO MAP, et al., 2021). Podemos identificar tais habilidades metacognitivas nos seguintes discursos:

"Ficamos nesse impasse de não conseguimos fazer aquela parte inicial, se não faz a parte inicial, não sabe fazer o final” (G).

"Semana passada na UTI, a médica pegou e falou bem assim: me dá um vasodilatador, e aí alguém gritou, ah, pega uma hidralazina para mim. Então na hora eu associei uma coisa com a outra e fui logo no vasodilatador para pegar a hidralazina" (I).

A HM de avaliação integra o CM e a EM para definir estado atual do resultado e curso futuro do processamento da tarefa. Acontece quando refletimos sobre o que ocorre depois de um evento ter acontecido, onde olhamos para o que fizemos e se isso levou ou não a um resultado. $E$ isso fica muito claro na fala de Gabriel, ao comentar a discrepância entre a conduta para vencer a olimpíada e a conduta correta para tratar a paciente. Isso porque naquele cenário específico havia um conjunto de ações que embora valesse pontos, não colaborava na conduta médica emergencial do caso dado o tempo reduzido que a equipe tinhapara atuar:

"Então, é bem assim: fazer o que o jogo espera da gente ou o que a gente sabe?" (R).

\section{DISCUSSÃO}

A análise do material coletado permitiu observar atividade metacognitiva variada. Para interpretá-la é conveniente referir-se ao longevo modelo de fluxo informacional metacognitivo proposto por Narens $L$ e Nelson TO (1990), que se mantendo atual por conta de sua simplicidadepermite interpretação clara dos dados aqui apresentados. Esses autores postulam o funcionamento da metacognição em dois níveis. $O$ primeiro inferior onde ocorre a Cognição. É o nível objeto. Neste é onde se localizam os fenômenos tradicionalmente atribuídos ao ato de conhecer e aprender, tais como ler, produzir esquemas e resumos, memorizar, apresentar e defender ideias seja pela palavra escrita seja pela fala. 
O segundo nível, conforme postulado por Narens L e Nelson TO (1990), localizado em posição lógica superior, situa-se a Metacognição. É o nível meta. Este estrato tem por função gerenciar o primeiro. Ali encontram-se os modelos ideais de funcionamento da cognição. É onde a mente "sabe" como usar a razão. Esta capacidade gerencial, fundamentada nos modelos ideais citados, executa suas funções por meio dos seus componentes, aqui descritos segundo Efklides A (2008). Neste estudo foram utilizadas as três categorias de maior nível descritas pela autora; Conhecimento Metacognitivo, Experiência Metacognitiva e Habilidade Metacognitiva. Já dito, o CM sendo o conhecimento declarativo sobre a EM e a HM. A EM relacionada ao sentimento do indivíduo durante as ações cognitivas. E finalmente as HM como as competências necessárias para o controle voluntário sobre seus próprios processos cognitivos.

Ainda mais, neste modelopara que a gerência metacognitiva possa ser exercida é necessário haver comunicação entre esses dois níveis. E ela se faz por meio de duas alças informacionais de retroalimentação recíproca. A alça de monitoração, de fluxo ascendente, informa ao nível meta o estado atual de funcionamento do nível objeto. De posse dessa informação, o nível meta, após processamento, fornece comandos ao nível objeto, por meio da alça descendente de controle (NARENS L e NELSON TO, 1990). E dessa forma, mostram os autores, fica o processo cognitivo mais eficiente, já que constantemente comparada a modelos ideais pode manter-se inalterado ou modificar-se em função da orientação fornecida pelo meta nível. E essa dinâmica mostrou-se claramente no processo de pensamento da equipe.

Não houve predominância significativa entre as categorias metacognitivas ( $C M=29, E M=22$ e $H M=26)$, fato que se sintoniza com a natureza do evento. Tratava-se de um contexto complexo, onde a equipe trabalhava sob pressão, solicitada (mesmo que simuladamente) a tomar decisões sobre a vida e a morte. $\mathrm{E}$ ainda mais, mesmo que simulado, os resultados de suas ações teriam efeitos muito reais em termos da autoestima e fracasso ou vitória na competição. Por isso não surpreende que todas as energias da equipe e aí incluídos os processos metacognitivos, estivessem sendo demandados ao máximo, individualmente ou em conjunto. Esse equilíbrio é ainda mais esperável na medida em que sabemos da necessária interação entre as categoriasassim como do papel gerenciador da metacognição sobre a cognição (EFKLIDES A, 2008; NARENS L e NELSON TO, 1994).

Durante a Olimpíada, o CM permitiu o uso de conhecimentos prévios armazenados na memória e o acionamento de estratégias que promoveram a monitorização e controle para melhor desempenho frente à atividade (DÍAZ-GUIO DA e ORTEGA FJ, 2019).

As HM tiveram dupla função. Por um lado, foram necessárias para o acesso ao $\mathrm{CM}$ para o fornecimento da matéria prima para a escolha e por outro para o uso em si da HM específica em um dado contexto. Neste sentido então é possível falar das $\mathrm{HM}$ em um relacionamento continuamente bidirecional entre os diferentes aspectos da habilidade e do conhecimento.

As habilidades mais observadas no estudo (monitoramento, controle, planejamento, avaliação, corregulação, memória de trabalho, reconhecimento de erros e resolução de conflito) foram acordes com a literatura, que as cita no contexto da simulação clínica. A aplicação das HM possibilitou a reflexão sobre a própria prática, conferindo aos participantes o necessário papel ativo na olimpíada, para agir e interagir com o contexto apresentado (MOREIRA MA, 2011).

O indivíduo que compreende a forma com qual ele aprende, amplia sua capacidade de construir seu conhecimento. E neste sentido prosseguem, apontando a Metacognição como ferramenta capaz de promover a consciência de si, favorecendo assim o processo de aprendizado. É justamente o processo de reflexão que leva o sujeito à tomada de consciência, ou seja, a apropriar-se das próprias ações. E ao apropriar-se de sua prática, ele constrói e/ou reconstrói as estruturas do seu pensar, ampliando sua capacidade de compreensão (BEBER B, et al., 2014; SILVA XC, et al., 2021).

Neste contexto, é razoável supor que a metodologia de simulação clínica, a partir da realidade recriada nos cenários propostos, pode ser capaz de fazer emergir o saber pensar do sujeito por meio dos seus processos metacognitivos e dessa forma, fornecer aos estudantes a condição de autonomia na tomada de decisão. 


\section{CONLUSÃO}

O presente estudo possibilitou desvelar sob a perspectiva metacognitiva, vertentes do pensamento humanoque de outras formas mais tradicionais permaneceram ocultos e, portanto, impermeáveis aos esforços docentes para o desenvolvimento de um estudante automotivado, reflexivo e autônomo. No entanto, este não é um estudo prescritivo. É antes de tudo descritivo. Não se pretende propor que os processos aqui, brevemente delineados, sejam os corretos ou mais adequados para o sucesso em qualquer situação similar. Neste momento o que se pode afirmar é a concorrência de formas de pensar, no momento entendidas como saudáveis, e o sucesso em uma competição. Por isso sugere-se o prosseguimento de pesquisas nesse campo.

\section{AGRADECIMENTOS}

À Comissão de Programação Científica por sua anuência à realização dessa pesquisa.

\section{REFERÊNCIAS}

1. ASSOCIAÇÃO BRASILEIRA DE EDUCAÇÃO MÉDICA (ABEM). Simulação em saúde para ensino e avaliação: conceitos e práticas, 2021. Disponível em: https://issuu.com/editoracubo/docs/issuu-abem. Acesso em outubro/2021.

2. BARDIN L. Análise de conteúdo. São Paulo: Edições 70, 2016; 141 p.

3. BEBER B, et al. Metacognição como processo da aprendizagem. Rev. psicopedag., São Paulo, 2014; 31(95): 144151.

4. BRANDÃO CFS, et al. Centros de simulação e projeto pedagógico: dois lados da mesma moeda. Scientia Medica, 2018; 28(1).

5. BRANDÃO CFS, et al. A simulação realística como ferramenta educacional para estudantes de medicina. Sci Med, 2014; 24(2): 187-92.

6. BRANNICK MT. Metacognition, OSCE performance anxiety and OSCE performance. Medical Education, 2013; 47(6): 540-542.

7. BRASIL. Resolução ํㅜ666, de 12 de dezembro de 2012. Dispõe sobre diretrizes e normas regulamentadoras de pesquisas envolvendo seres humanos. Diário Oficial [da] República Federativa do Brasil, Brasília, DF,13 jun. 2013.

8. BRASIL. Resolução ํㅜ 510, de 07 de abril de 2016. Dispõe sobre as normas aplicáveis a pesquisas em Ciências Humanas e Sociais. Diário Oficial [da] República Federativa do Brasil, Brasília, DF, 24 maio 2016.

9. CARVALHO AB, et al. Análise de conteúdo de duas avaliações externas brasileiras de cursos de medicina: Enade e Revalida. Rev. bras. educ. med, 2021; 45(1): 1-10.

10. CARVALHO DRS, et al. Simulação em saúde: história e conceitos cognitivos aplicados. Rev Inter Educ Saúde. 2021; 5(1): $1-12$.

11. CAVEIÃO C, et al. Tendências e estratégias de ensino-aprendizagem utilizadas no desenvolvimento da liderança do enfermeiro. Revista Brasileira de Enfermagem, 2018; 71(1): 1531-1539.

12. LINS ML, et al., Estratégias para ensinar liderança na graduação de enfermagem. Rev Rene, 2018; $19(1)$ : 1-8.

13. DÍAZ-GUIO DA, RUIZ-ORTEGA FJ. Relationship among mental models, theories of change, and metacognition: structured clinical simulation. ColombianJournalofAnesthesiology. 2019; 47(1): 113-116.

14. DREIFUERST KT. Getting Started With Debriefing for Meaningful Learning. Clinical Simulation in Nursing, 2015; 11(5): 268-275.

15. EFKLIDES A. Metacognition: Defining its facets and levels of functioning in relation to self-regulation and coregulation. EuropeanPsychologist, 2008; 13(4): 277-287.

16. EFKLIDES A, et al. Feeling of difficulty: An aspect of monitoring that influences control. European Journal of Psychology of Education, 1999; 14(4), 461-476.

17. FLATO UAP, GUIMARÃES HP. Educação baseada em simulação em medicina de urgência e emergência: a arte imita a vida. Revista Brasileira Clínica Médica. São Paulo, 2011;9(5):360-364.

18. FLAVELL JH. Metacognition and cognitive monitoring: A new area of cognitive-developmental inquiry. American psychologist, 1979; 34(10): 900-906.

19. FLAVELL JH. Metacognitive aspects of problem solving. The nature of intelligence, 1976.

20. GOMES TR, et al. O erro médico sob o olhar do Judiciário: uma investigação no Tribunal de Justiça do Distrito Federal e Territórios. Cadernos Ibero-Americanos de Direito Sanitário, 2017; 6(1): 72-85. 
21. JONES F, et al. Simulation in Medical Education: Brief history and Methodology. Principles and Practice of Clinical Research. 2015; 2(1): 56-63.

22. KANEKO RMU, LOPES MHBM. Realistic health care simulation scenario: what is relevant for its design? RevEscEnferm USP. 2019; 53(1):e03453.

23. LOCATELLI SW. Tópicos de Metacognição: para aprender e ensinar melhor. Curitiba: Appris, 2014. 77p.

24. MENDONÇA V, et al. Erro médico: conceito, caracterização e gestão. Saúde e Sociedade, 2019; 28(4): $255-266$.

25. MINAYO MCS. Pesquisa social: teoria, métodos e criatividade. 29ae ed. Petrópolis, RJ: Vozes, 2010, 144p.

26. MOREIRA MA. Teorias de aprendizagem. 2. ed. São Paulo: EPU, 2011.

27. NARENS L, NELSON TO. Why Investigate Metacognition? In: METCALFE, J.; SHIMAMURA, A. P. (Eds.). Metacognition. [s.I.] The MIT Press, 1994: 1-25.

28. PATIAS ND, HOHENDORFF JV. Critérios de qualidade para artigos de pesquisa qualitativa. Psicol. estud., 2019; 24(1): e43536..

29. PEIXOTO MAP, et al. Construção de definições operacionais em Metacognição. 2021.

30. PEIXOTO MAP, et al. Usando a metacognição para analisar um caso de erro diagnóstico em simulação de alta fidelidade. Revista Brasileira de Educação Médica, 2021; 45(2): e080.

31. ROSA RR, et al. Efeitos do xadrez nas funções cognitivas e na aprendizagem de escolares: uma revisão sistemática. Research, Society and Development, 2020; 9(6).

32. SILVA XC, et al.Metacognição e suas ferramentas para o aprendizado. Revista Eletrônica Debates em Educação Científica e Tecnológica, 2021; 10(2).

33. SINZ E, et al. Reliability of simulation-based assessment for practicing physicians: performance is context-specific. BMC Medical Education; 2021; $21: 207$.

34. TEIXEIRA CRS, et al. Avaliação dos estudantes de enfermagem sobre a aprendizagem com a simulação clínica. Revista Brasileira de Enfermagem, 2015; 68(1): 311-319. 